\title{
News article consumption habits of Greek internet users
}

\author{
Evangelia Avraam ${ }^{1}$ \\ Andreas Veglis ${ }^{2}$ \\ Charalampos Dimoulas ${ }^{3}$
}

\begin{abstract}
The concept of different news consumption habits during a day has been well known for many decades in the broadcasting industry. News websites are also experiencing a drop in late afternoon traffic and a sharp drop in the evening hours. Furthermore, during the weekend, website traffic numbers appeared to be significantly different than the numbers during the weekdays. That resulted in the adoption of the concept of dayparting in the case of the internet. The existence of internet dayparts can have a significant impact on news websites since they can significantly determine their success. It is quite natural to assume that media organizations have adopted their publishing patterns to best satisfy the audience's consumption patterns. This paper investigates those consumption patterns by conducting an extensive web survey among university students and particularly journalism and communication students that are expected to exhibit high consumption rates. The parameters being investigated include time, weekdays and weekends, and content categories. The study identified distinct periods that exhibit specific consumption patterns. The results appear to be to some degree in agreement with findings of previous studies that reported on publishing patterns, but significant differences have also been identified. Those results can provide valuable information for the implementation of successful content publishing strategies from the media organizations.
\end{abstract}

Keywords: consumption patterns, dayparts, web news article, media.

\footnotetext{
${ }^{\mathbf{1}}$ PostDoc Researcher, Electronic Media Lab, School of Journalism and Mass Communications, Aristotle University of Thessaloniki, Greece, e-mail: avraam@jour.auth.gr

${ }^{2}$ Professor, Media Informatics Lab, School of Journalism and Mass Communications, Aristotle University of Thessaloniki, Greece, e-mail: $\quad$ veglis@jour.auth.gr

${ }^{3}$ Associate professor, Electronic Media Lab, School of Journalism and Mass Communications, Aristotle University of Thessaloniki, Greece, e-mail: babis@jour.auth.gr
} 


\section{INTRODUCTION}

The digitalization of journalism during the last 10 years of the previous century has completely transformed the way news is generated and disseminated to the audience (Siapera and Veglis, 2012). The internet along with its services has offered alternative dissemination channels for the news organizations (www, RSS, web-push notifications, mobile apps, social networking, microblogging to name some of them) (Veglis, 2012). This novel medium landscape has enabled media organizations to target different audience groups that exhibit specific news consumption characteristics (consumption time, mobile/home user, device type, etc) (Taneja et al. 2012, Avraam et al. 2021). That resulted in the complete transformation of the media industry, where new players (Facebook, Google) have emerged and control to a significant extent the news that the general public receives (Brake, 2017; Kolodzy, 2006).

In this context and taking into account, the fears competitions among media organizations to attract the audience (Perego, \& Yuksel, 2018), understanding the news consumption habits of the internet users becomes very crucial (Wolf \& Schnauber, 2015). In nowadays the continued publication of news 24 hours a day is an established norm. News concerning break events is posted almost immediately on various publication channels (Avraam et al, 2021). This means that media organizations are forced to combine "real-time" adaptation of events, audience personalization preferences, and media specialization (American Press Institute, 2014). This is accomplished through the analysis of statistical data which determine the web traffic of the media organization. The aim is to optimize the services provided to the audience. Of course, the above practices also aim at increasing fame and recognition, establishing credibility, and building relationships of trust with the audience, thus increasing revenues (Colace, Casaburi, De Santo \& Greco 2015; Elliott, Chuma, Gendi, Marko \& Patel 2016; Leskovec 2011; Pang \& Lee 2008; Schultz, Block \& Viswanathan 2016; Spann, Molitor \&Daurer 2016). The problem is that the news consumption from many publication channels is not easy to measure with software automation. Thus, direct audience surveys are needed to be utilized to measure actual news consumption from the audience (Thorson, 2008; Yuan, 2011).

This paper attempts to find news consumption patterns by utilizing a web survey among university students and particularly journalism and communication students. The latter are expected to exhibit high consumption rates because news is part of their discipline under study. The parameters being investigated include time, weekdays and weekends, type of content, and content categories.

The rest of the paper is organized as follows. Section 2 includes a literature review on news consumption. This is followed by the methodology section in which information about the methodology employed in this study is discussed. Section 4 presents the results of the survey. Next, the results are discussed, and a dayparting model is proposed. Concluding remarks, as well as future extensions of this work, are included in the last section. 


\section{LITERATURE REVIEW}

Content consumption and the existence of consumption patterns have been investigated quite thoroughly in the broadcast industry (Beyers, 2004). The concept of dayparting was introduced, which can be broadly defined as a consecutive block of time on similar days (weekdays or weekends) during which, the size of the audience is homogeneous as is the characterization of the group using the medium (OPA, 2003; Veglis, 2014). These segments have a distinct audience as far as their preferences are concerned, something that works well for some advertisers but not so well for others. Each daypart on radio and television has its personality, which is reflected in its programming and advertising (MORI Research, 2003). In other words, in the broadcast industry, the researchers have identified specific consumption patterns that were exploited to increase the audience and also advertisement revenues.

But although the concept of dayparting was well known and well investigated in the broadcast industry, it was not anticipated, at least at the beginning, that it will also be present in the case of WWW. This was to some extent understandable since the web news articles are always available on the internet so there is no reason to study when the actual consumption takes place (Veglis, 2014). Thus, media planning for web content was only interested in overall site traffic, demographics, and content affinity and not for the dynamic changes of the audience over time.

All this changed in 2020 when two studies indicated specific consumption patterns among internet users in the USA (Beyers, 2004). The first one was conducted by MORI for the NAA (Newspaper Association of America (MORI, 2003) and confirmed specific consumption patterns by online newspaper readers. The second one was supervised by the OPA (Online Publishers Association) and identified five district dayparts, which differ in usage levels, demographics, and type of consumed content (2003). Similar results were reported five years later by Burst Media that studied the internet habits of women above 25 years old. The following year Newell et al. published a study on media consumption which compared new media usage with other traditional media (newspapers, magazines, radio, and television). In 2010 BARI identified four distinct dayparts in the consumption habits of Greek online newsreaders. Finally, Veglis (2014) reported the existence of dayparts based on traffic data from Greek media websites.

Various Internet dayparts were proposed in each of the surveys presented in the literature review. On weekdays, OPA identified four dayparts and one daypart on weekends. MORI did no distinguish between weekdays and weekends and reported four dayparts. Newell et al, identified six dayparts, each with a short length. Bari (2010) divided the day into four equal dayparts, while Burst Media (2007) used five. Avraam (2012) took Bari's dayparts as a starting point and suggested minor changes based on the Greek workday. Finally, Veglis (2014) proposed four dayparts, with no distinction between workdays and weekends. 
Table 1: Definition of dayparts in various studies

\begin{tabular}{|c|c|c|c|}
\hline time period & Burst Media (2007) & Newell et al. (2008) & Veglis (2014) \\
\hline $00.01-01.00$ & \multirow{7}{*}{ Early morning } & \multirow{6}{*}{ Overnight } & \multirow{6}{*}{ Night } \\
\hline $01.01-02.00$ & & & \\
\hline $02.01-03.00$ & & & \\
\hline $03.01-04.00$ & & & \\
\hline 04.01-05.00 & & & \\
\hline $05.01-06.00$ & & & \\
\hline $06.01-07.00$ & & \multirow{4}{*}{ Morning drive } & \multirow{4}{*}{ Morning } \\
\hline $07.01-08.00$ & \multirow{5}{*}{ Morning-noon } & & \\
\hline $08.01-09.00$ & & & \\
\hline $09.01-10.00$ & & & \\
\hline $10.01-11.00$ & & \multirow{3}{*}{ Mid-day } & \multirow{7}{*}{ During the day } \\
\hline $11.01-12.00$ & & & \\
\hline $12.01-13.00$ & & & \\
\hline $13.01-14.00$ & Afternoon-noon & \multirow{3}{*}{ Afternoon - noon } & \\
\hline $14.01-15.00$ & \multirow{5}{*}{ Late afternoon } & & \\
\hline $15.01-16.00$ & & & \\
\hline $16.01-17.00$ & & \multirow{2}{*}{ Afternoon drive } & \\
\hline $17.01-18.00$ & & & \multirow{7}{*}{$\begin{array}{l}\text { Afternoon- } \\
\text { evening }\end{array}$} \\
\hline 18.01 .19 .00 & & \multirow{5}{*}{ Primetime } & \\
\hline $19.01-20.00$ & \multirow{5}{*}{ Evening } & & \\
\hline $20.01-21.00$ & & & \\
\hline $21.01-22.00$ & & & \\
\hline $22.01-23.00$ & & & \\
\hline $23.01-00.00$ & & Overnight & \\
\hline
\end{tabular}

The exact daypart models of three of the most recent studies are presented in table 1. All studies propose a similar number of dayparts (4 to 6). The most recent model suggests dayparts with comparable time durations (Veglis, 2014). On the other hand, the other two studies include dayparts that exhibit significant variation in size. The model of Newell et al. (2008) includes two dayparts that are related to daily activities (morning drive, afternoon drive) that can be considered as country/culture-specific. 


\section{METHODOLOGY}

This research was funded by Greece and the European Union (European Social Fund) through the Operational Program "Human Resources Development, Education and Lifelong Learning", in the framework of the Act "SUPPORT OF POSTGRADUATE RESEARCHERS - B cycle" (MIS 5033021) implemented by the State Scholarships Foundation

(IKY). In order to explore the consumption habits of web news consumers, a web survey was conducted. The participants were journalism and communication university students. The selection of this particular group was based on the fact that young people exhibit a very high percentage of internet usage and due to their field of study this particular group shows a high interest in news consumption. Also due to the nature of their study, they are considered to be above average computing devices users. The survey was conducted from $15^{\text {th }}$ to $25^{\text {th }}$ of May. The survey tool was a web questionnaire that included 15 close-ended questions and it was deployed on the Lime Survey platform of the School of Journalism and Mass Communication of Aristotle University of Thessaloniki (https://surveys.jour.auth.gr/). The dissemination of the web questionnaire was done through the school's official mailing lists, through the university's e-learning platform (https://elearning.auth.gr). It is also worth mentioning that during the survey preparation, all ethical approval procedures and rules suggested by the "Committee on Research Ethics and Conduct" of the Aristotle University of Thessaloniki were followed.

The number of participants in the survey was 171, 153 questionnaires were completed and 18 were partially answered. Some demographic data for the participants $73 \%$ were female and $27 \%$ male. The majority of the participants $83 \%$ belong to the $18-27$ age group and the second larger age group was the 28-37 age group with $11 \%$. The rest of the participants were older. As far as education level is concerned $77 \%$ reported to be university students and the majority of the rest of the participants were also university students at the post-graduate level. The complete data can be found in Table 2 .

\section{Table 2: Education level of the participants.}

\begin{tabular}{|l|c|}
\hline Education level & percentage \\
\hline Graduate of secondary education & $1 \%$ \\
Higher education student & $77 \%$ \\
BA graduate & $2 \%$ \\
MA student & $11 \%$ \\
MA graduate & $5 \%$ \\
Phd student & $1 \%$ \\
Phd holder & $3 \%$ \\
\hline
\end{tabular}




\section{RESULTS}

Since the sample of the participants were journalism and communication university students, it was expected that they will be closed related to the use of technology. Almost all the participants own or use a laptop computer and a smartphone. It is worth noting that smartphone usage is slightly higher than the one of the laptop computers. Roughly half of the participants own or use a desktop computer and only $39 \%$ own or use a tablet (see figure 1). This finding confirms the initial assumption that journalism and communication students are above average computing device users.

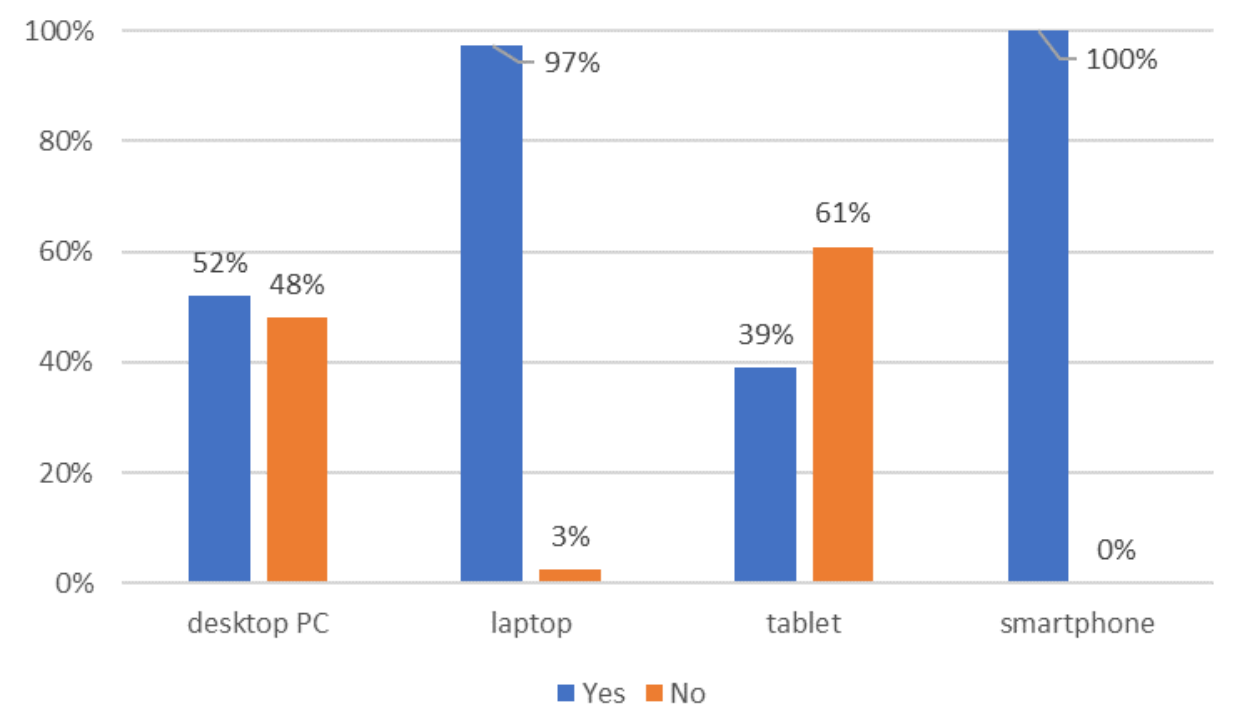

Figure 1: Percentages of ownership or usage of computing devices.

Social networking services are frequently used by internet users around the world for staying informed about current news (Boukes, 2019). The participants of the survey were asked to state the frequency usage of the most widely used social networking services (Facebook, Twitter, Instagram, and YouTube). The results show that Instagram is the most frequently used service with $26 \%$ of the participants reporting that they are always using it. Second is YouTube and Facebook appears to be third. Twitter appears to be the less popular social networking service with more than half of the participants replying that they never use it. This is quite an interesting result since Twitter is considered to be the main channel for news dissemination (Seth, et al., 2017). Nevertheless, the majority of the participants report on using Facebook, Instagram, and YouTube at least every day. All data concerning frequency usage of social networking services are displayed in figure 2. 


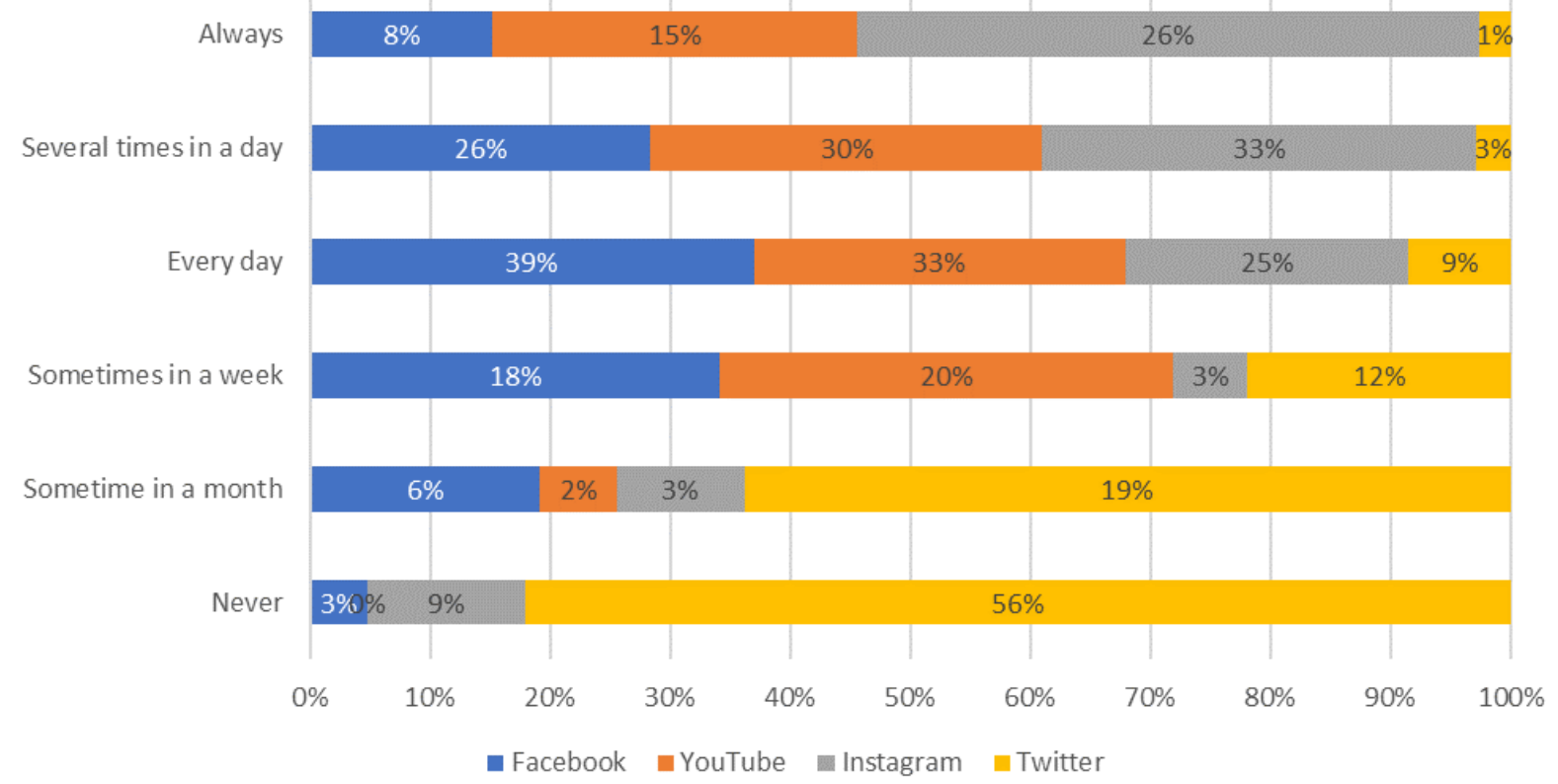

Figure 2: Frequency usage of social networking services (Instagram, YouTube, Facebook, and Twitter).

Next, the participants were asked to rate on a Likert scale (1-5) the sources they utilize to stay up to date with current news. Media websites appear to be the first choice of the responders with media organizations' social accounts a close second. On the other hand, in the third place, it was found to be social media accounts of friends. In other words, social networking services are utilized by the participants, but the media organizations' accounts are higher in preference than the accounts of friends. Based on that finding we can conclude that the participants utilize social media accounts to stay informed, but they exhibit a strong preference for official media organization accounts, which is an important factor in dealing with the problem of fake news (Katsaounidou, Dimoulas, \& Veglis, 2018). It is worth noting that media organizations' apps (for mobile devices) do not attract a significant percentage of preferences (see figure 3). This may be explained by the fact that media organizations in Greece have developed apps with very basic features.

Moving to the category of news participants are interested in, participants we asked to rate on a Likert scale (1-5) 10 different thematic categories on news. Society news was found to be the most popular category with sports the least popular. It is worth reminding that $73 \%$ of the participants were female that are usually not very keen on sports news and this might explain the previous finding of the survey. International and health (due to the COVID-19) and culture news exhibit high interest. Figure 4 includes the classification of all 10 news categories. 


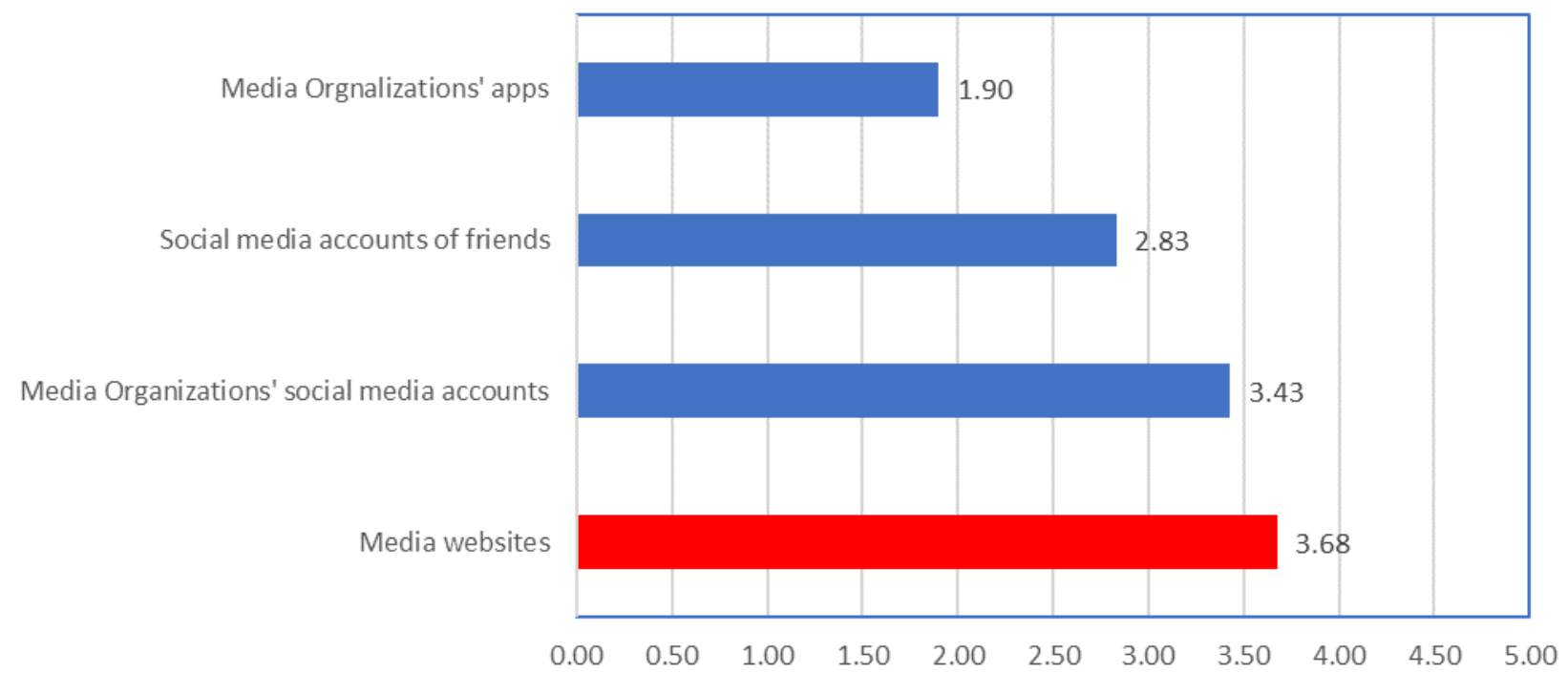

Figure 3: Methods for staying informed on current news (Likert scale 1 to 5).

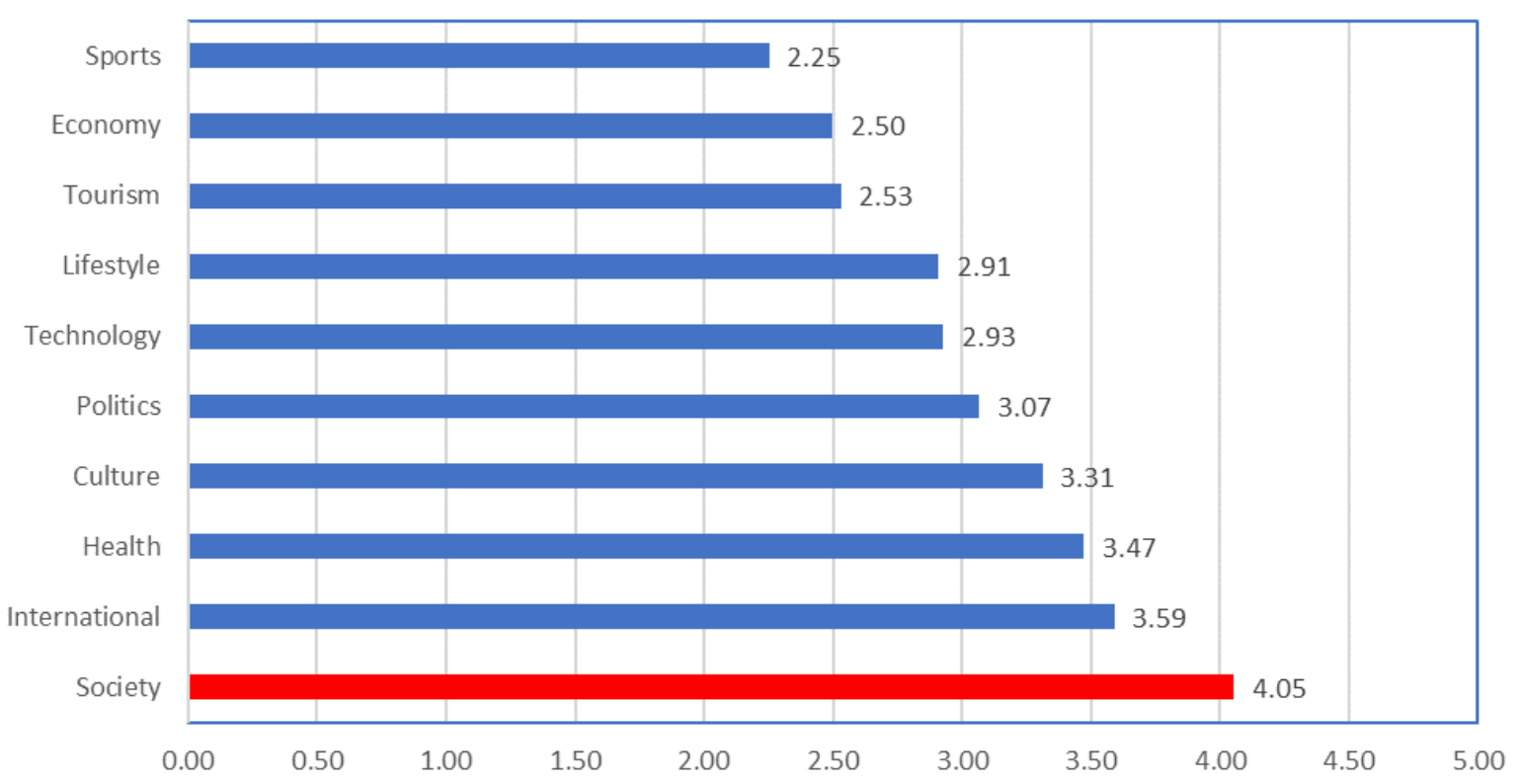

Figure 4: Consumption rates of different categories of news (Likert scale 1 to 5 ).

Next, the news-consumption behavior of the participants throughout the week was investigated. Specifically, the participants were asked to rate on a Likert scale (1-5) the probability of consuming the news each day of the week. Previous studies were reported a significant decrease in the readership during the weekend (Avraam et al, 2021; Veglis, 2012). This result was also found in our survey. In other words during weekends participants rated news consumption at an average of 3.35, while during the weekdays the average rate was 3.61 (rouphly an $8 \%$ decrease). During the weekdays the rate fluctuates between 3.68 and 3.54. It is worth noting that Monday appears to be the weekday during which participants seem to be more eager to read the news while Tuesday is the day during which the 
participants appear to be less interested in staying informed. Also, Friday is the weekday with the second highest news consumption rate. Nevertheless, it is worth noting that the differences during the weekdays and during weekdays and weekends appear to be quite small. All the previously mentioned results are included in figure 5.

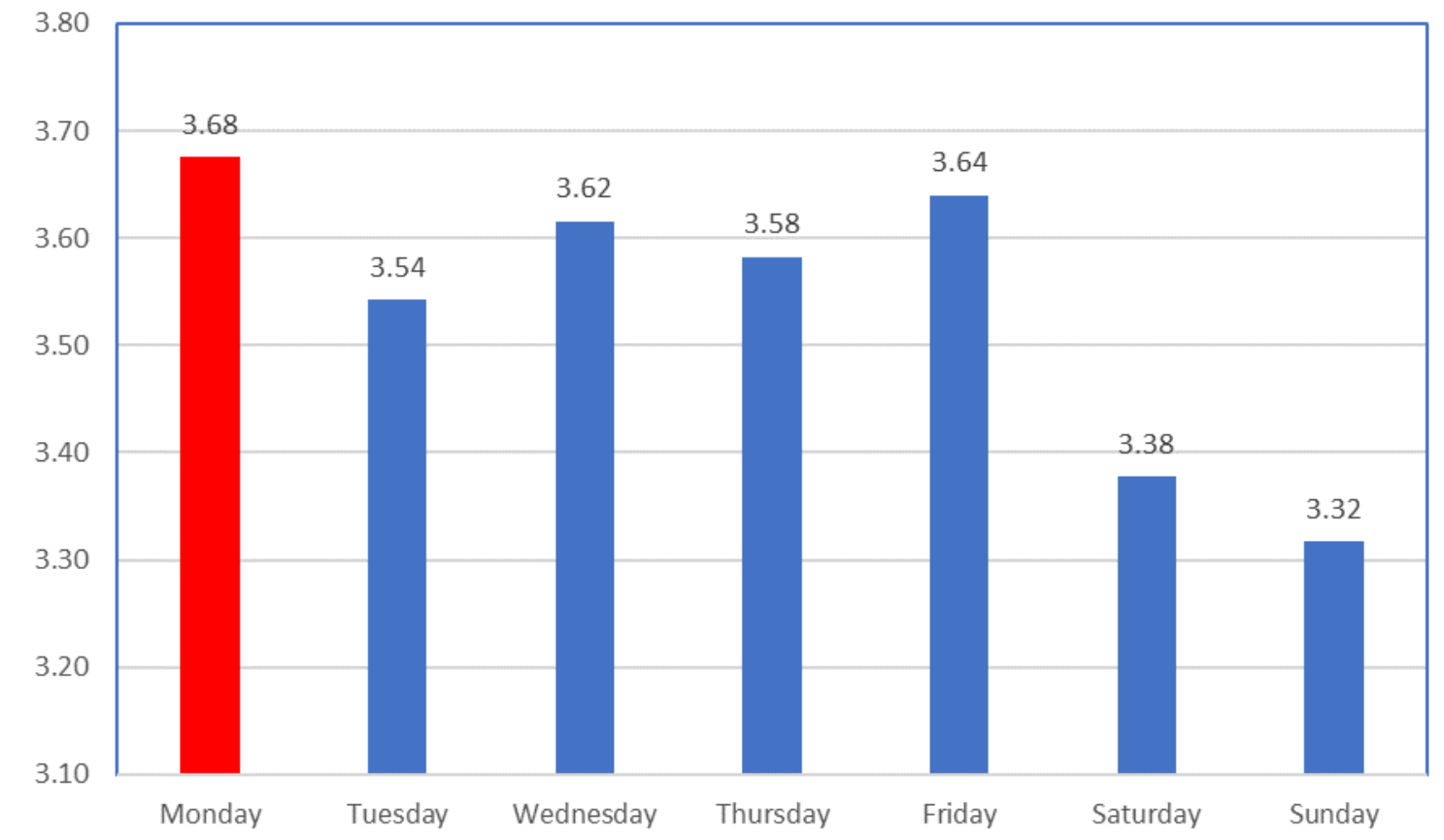

Figure 5: Consumption rates during the days of the week (Likert scale 1 to 5).

The last topic that was investigated was the participants' consuming behavior throughout the 24 time period of a day. The day was divided into nine 2-hour time slots starting from 06.01 until midnight while an additional time slot was assigned to the 00.01- 06.00 time period. This choice was made to limit the time slots to some extent, thus facilitating in this way the entry of data by the survey participants. The results indicate that there is a significant change in the news consumption rate during the 24 hours of the day. Specifically, there is a $58 \%$ difference between the lower rate which is exhibited during the 06.01-08.00 time slot, and the 20.01-22.00 time slot, during which, the highest interest for news consumption is demonstrated from the participants. Although the highest value is exhibited quite late, it is worth noting that from 10.01 up to 00.00 (for 12 consecutive hours) the interest in news consumption remains high and almost constant. Another finding that is worth stressing is the fact that the lower consumption interest is to be reported during the 00.01-06.00 time period, but during the early morning period. This can be explained by examining the composition of the participants, where the majority of them are students that tend to go to sleep at the early hours of the day and wake up a bit late (Buboltz et al., 2009). Another interesting finding is the period than the highest news consumption rate is exhibited. Especially in Greece during this period, the majority of the national coverage TV stations broadcast their main news bulleting (Maniou, 2013), although in recent years there seems to be a tendency to broadcast 
DOI https://doi.org/10.34097/jeicom-3-2-december2021-5

CC BY

earlier. This fact may be related to the above findings since this period is strongly related to learning the current news.

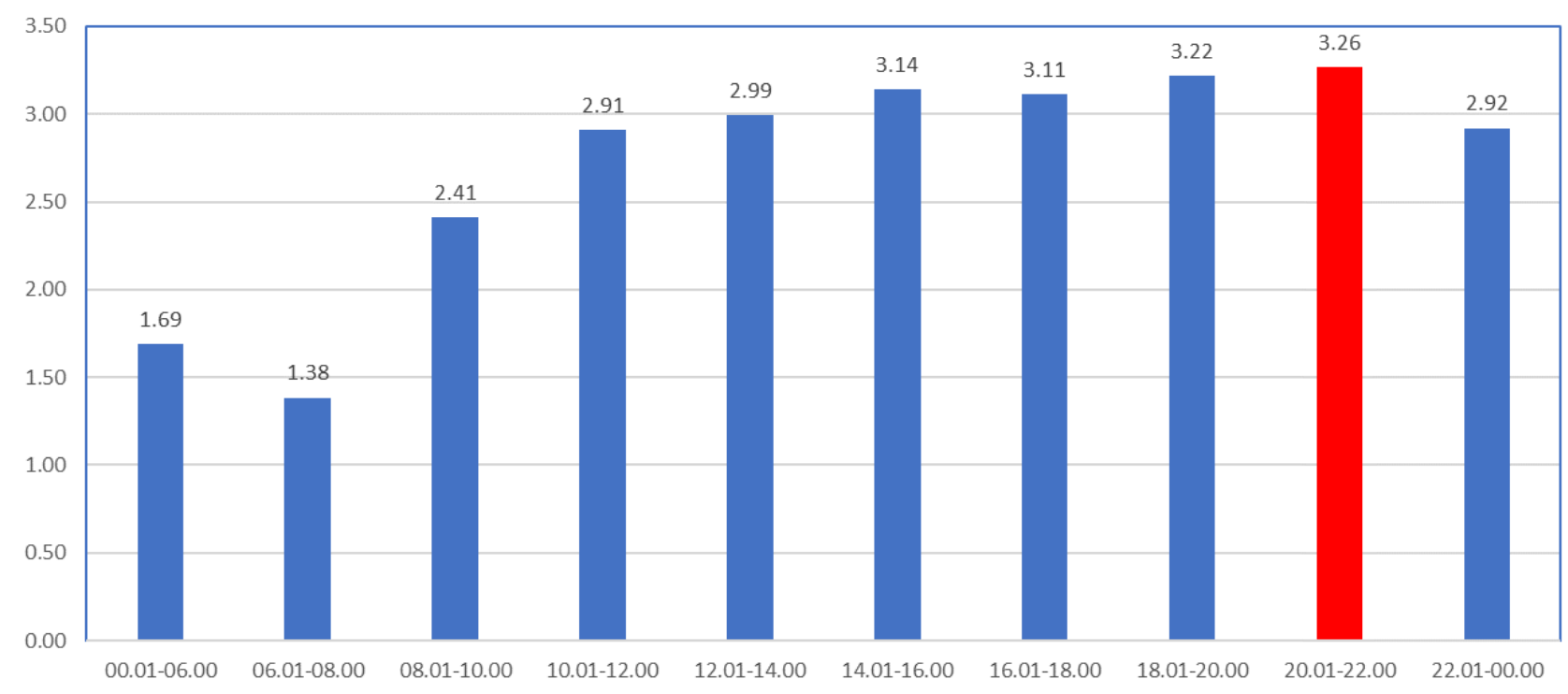

Figure 6: Consumption rates during different periods in 24 hours (Likert scale 1 to 5).

\section{DISCUSSION}

Based on the results presented in the previous section it can be concluded that it is possible to identify distinct periods that exhibit different consumption patterns. The first thing that needs to be decided is whether weekdays and weekends should be considered as periods that exhibit different characteristics. Although weekends display a decreased rate of interest in news consumption, the existing data, and taking into account the relatively small drop of interest, do not justify proposing the distinction between weekdays and weekends. More specific related data needs to be collected to identify distinctive characteristics (category of news, consumption method, etc.) that weekends and weekends may have.

Moving to the issue of determining dayparts in the news consumption during 24 hours, things appear to be clearer. Specifically, based on the findings the period from 06.01 to12.00 displays a significant increase in the news consumption interest. During the period 12.01 to 22.00 , the consumption rate does not appear to change considerably. It is worth mentioning that during this period the highest news consumption rate is located. Finally, from 22.01 until 8.00 in the morning there is a significant drop in the news consumption rate. Based on the previous analysis the study proposes a news consumption model that is comprised of three dayparts, namely morning (06.01-12.00), day (12.01-22.00), and night (22.01-8.00).

The proposed dayparts along with the data from figure 6 are displayed in figure 7 . 


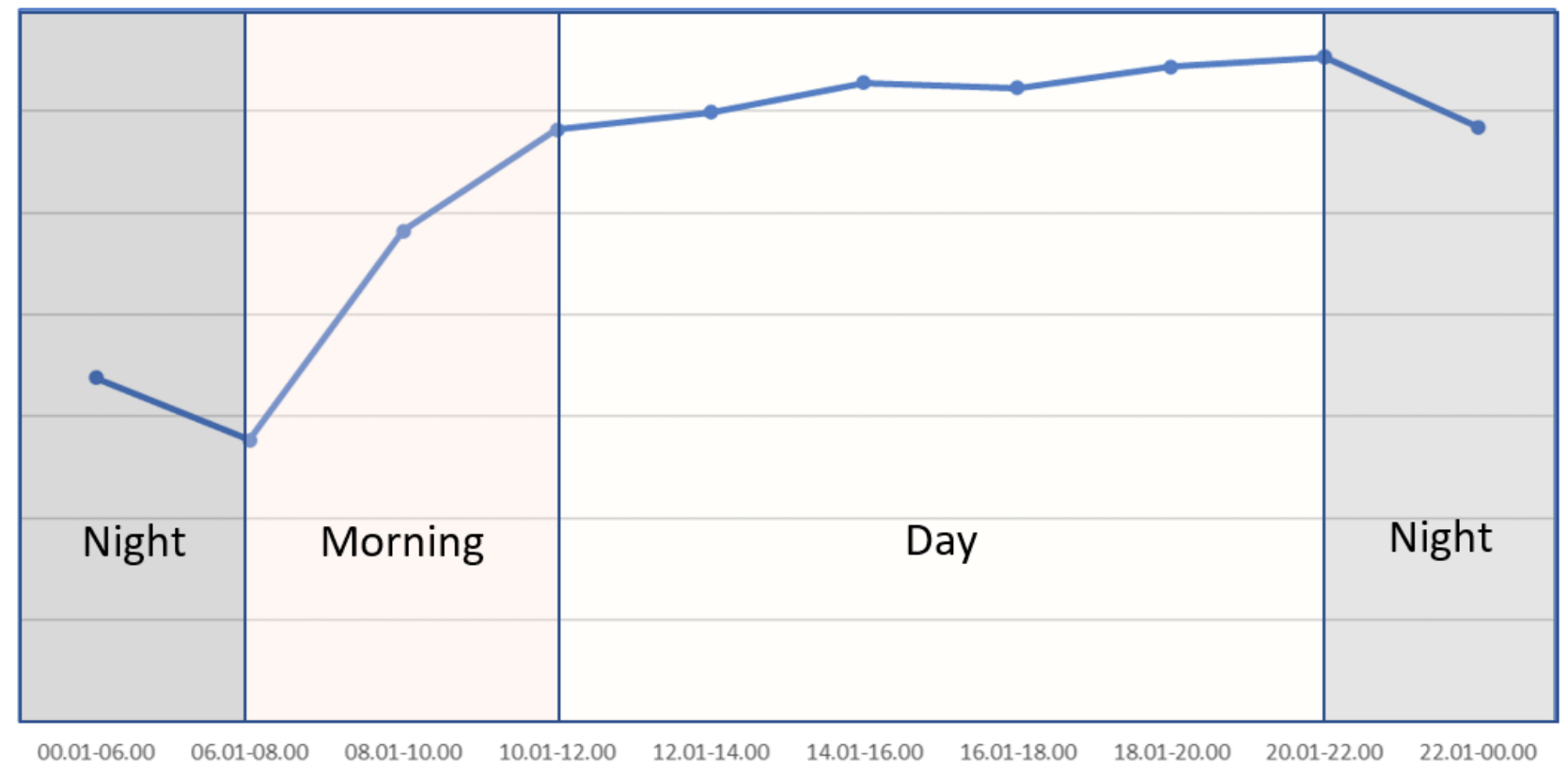

Figure 7. The proposed daypart model in comparison with the consumption data collected through the survey.

The proposed dayparting model includes only three dayparts in comparison with the 4-6 dayparts of the past proposed models. That makes it simpler to understand and use. In the last decade, there were significant changes in technology and the services people use to stay informed. The smartphone is one of the most important parameters that allow users to have direct access to information wherever they are. Thus, various dayparts that were associated with commuting, during which the news consumption was decreased are not applicable today. Also, the use of social networking services can notify users instantly about breaking news is now common practice (Veglis, 2012). This gradual shift to simpler dayparting models is also evident from Table 1 since the older a model is the greater number of dayparts it includes. 


\section{CONCLUSIONS}

This paper has investigated the consumption habits of Greek internet users. Specifically, the study attempted to identify dayparts that are segments of time during which the audience exhibits homogeneous news consumption characteristics. The study adopted an online survey as us main data collection tool that was conducted among journalism and communication university students. This particular group is very interested in current events and are considered to be above average computing devices users. The findings of the study supported the formulation of a dayparting model that is comprised of three time zones, namely morning (06.01-12.00), day (12.01-22.00), and night (22.01-8.00). Also, the findings did not detect significant consumption patterns between weekdays and weekends, except for a small decrease in the interest in news consumption. One interesting characteristic of the proposed dayparting model is its simplicity since it utilized only three dayparts. The results of the study can be proven valuable to media organizations since they can fine-tune their publishing program to match the users' news consumption habits, thus maximizing the teaching of their news products and increasing their revenues.

This study has also certain limitations. The number of female participants in the survey is 3 times the number of male participants, a factor that may influence the obtained results. Also, the participants of the study are mainly young people belonging to the 18-27 age group.

Future extensions of this work may involve a broad sample as far as age is concerned and also a more detailed study of other factors that may influence consumption patterns, like device used and utilization of specific dissemination channels (web, social, app, etc.).

\section{ACKNOWLEDGMENTS}

This research was funded by Greece and the European Union (European Social Fund) through the Operational Program "Human Resources Development, Education and Lifelong Learning", in the framework of the Act "SUPPORT OF POSTGRADUATE RESEARCHERS - B cycle" (MIS 5033021) implemented by the State Scholarships Foundation (IKY). 


\section{REFERENCES}

American Press Institute. 2014. Social and demographic differences in news habits and attitudes. Available at: https://www.americanpressinstitute.org/publications/reports/survey-research/social-demographicdifferences-news-habits-attitudes/

Avraam, E. (2012). Contribution of new information and communication technologies in the production distribution and consumption of news (in Greek). Phd dissertation, School of Journalism \& MMC, Aristotle University of Thessaloniki, Greece.

Avraam, E., Veglis, A., \& Dimoulas, C. (2021). Publishing Patterns in Greek Media Websites. Social Sciences, 10(2), 59.

Beyers, H. (2004). Dayparting online: living up to its potential?. International Journal on Media Management 6.1-2: $67-73$

Boukes, M. (2019). Social network sites and acquiring current affairs knowledge: The impact of Twitter and Facebook usage on learning about the news. Journal of Information Technology \& Politics, 16(1), 3651.

Brake, D. R. (2017). The invisible hand of the unaccountable algorithm: How Google, Facebook and other tech companies are changing journalism. In Digital Technology and Journalism (pp. 25-46). Palgrave Macmillan, Cham.

Buboltz Jr, W., Jenkins, S. M., Soper, B., Woller, K., Johnson, P., \& Faes, T. (2009). Sleep habits and patterns of college students: an expanded study. Journal of College Counseling, 12(2), 113-124.

Burst Media. (2007). Online Insights, June.

Colace, F., Casaburi, L. De Santo, M., and Greco, L., (2015). Sentiment detection in social networks and in collaborative learning environments. Computers in Human Behavior 51:1061-1067.

Elliott, C., Chuma, W., Gendi, Y. E., Marko, D., \& Patel, A. (2016). Hate Speech, Key concept paper in Media, Conflict and Democratisation (MeCoDEM).

Katsaounidou, A., Dimoulas, C., \& Veglis, A. (Eds.). (2018). Cross-media authentication and verification: emerging research and opportunities. IGI Global.

Kolodzy, J., (2006). Convergence journalism: Writing and reporting across the news media. Rowman \& Littlefield.

Leskovec, J. (2011). Social media analytics: tracking, modeling and predicting the flow of information through networks. In Proceedings of the 20th international conference companion on World wide web (pp. 277278).

Maniou, T. (2013), Television, society and Political News (in Greek), Epikentro.

MORI Research. (2003). Online Dayparting: Claiming the Day, Seizing the Night, Report.

OPA (2003). The Existence and Characteristics of Dayparts on the Internet. The OPA White Papers, January.

Pang, Bo., Lee, Lillian. 2008. Opinion mining and sentiment analysis. Foundations and Trends® in Information Retrieval 2(1-2):1-135.

Perego, J., \& Yuksel, S. (2018). Media competition and social disagreement. Work.

Schultz, D. E., Block, M. P., \& Viswanathan, V. (2018). Consumer-driven media planning and buying. Journal of Marketing Communications, 24(8), 761-778. 
Journal of Education, Innovation and Communication, sponsored by the Communication Institute of Greece

DOI https://doi.org/10.34097/jeicom-3-2-december2021-5

CC BY

Seth, A., Nayak, S., Mothe, J., \& Jadhay, S. (2017). News Dissemination on Twitter and Conventional News Channels. In 19th International Conference on Enterprise Information Systems (ICEIS 2017) Vol. 1, pp. pp-43.

Spann, M., Molitor, D., \& Daurer, S. (2016). Tell Me Where You Are and I'll Tell You What You Want: Using Location Data to Improve Marketing Decisions. NIM Marketing Intelligence Review, 8(2), 30-37.

Siapera, E., and Veglis, A., (eds) (2012). The handbook of global online journalism. John Wiley \& Sons.

Taneja, H., Webster, J. G., Malthouse, E. C., \& Ksiazek, T. B. (2012). Media consumption across platforms: Identifying user-defined repertoires. New media \& society, 14(6), 951-968.

Thorson, E., (2008). Changing patterns of news consumption and participation: News recommendation engines. Information, Communication \& Society, 11(4), pp.473-489.

Wolf, C., \& Schnauber, A. (2015). News consumption in the mobile era: The role of mobile devices and traditional journalism's content within the user's information repertoire. Digital journalism, 3(5), 759776 .

Veglis, A. (2014). Dayparting in online media: The case of Greece. International Journal of Computers and Communications 8:77-85.

Veglis, A. (2012). Journalism and Cross-Media Publishing: The Case of Greece. In The Handbook of Global Online Journalism (eds E. Siapera and A. Veglis):209-230.

Yuan, E., (2011). News consumption across multiple media platforms: A repertoire approach. Information, Communication \& Society, 14(7), pp.998-1016. 
Journal of Education, Innovation and Communication, sponsored by the Communication Institute of Greece

DOI https://doi.org/10.34097/jeicom-3-2-december2021-5

CC BY

This Journal Article is brought to you for free and open access by the double blind reviewed Journal of Education, Innovation and Communication, sponsored by the Communication Institute of Greece. For more information, please take a look at https://coming.gr/journal-of-education-innovation-and-communicationjeicom/ or/and email us at jeicom@coming.gr 